\title{
Estructura factorial del Cuestionario de Experiencias Relacionadas con Internet en universitarios chilenos
}

\author{
Factorial structure of the Questionnaire of Experiences Related with Internet \\ on Chilean university students
}

Tristán Inostroza ${ }^{1}$, Felipe Madrid $^{2}$, Manuel Salinas ${ }^{3}$, Evaristo Reyes ${ }^{4}$ y Cristóbal Guerra $^{5}$
${ }^{1}$ Psicólogo. Escuela de Psicología, Facultad de Ciencias Sociales y Comunicaciones, Universidad Santo Tomás, Chile.
${ }^{2}$ Psicólogo. Escuela de Psicología, Facultad de Ciencias Sociales y Comunicaciones, Universidad Santo Tomás, Chile.

${ }^{3}$ Psicólogo. Escuela de Psicología, Facultad de Ciencias Sociales y Comunicaciones, Universidad Santo Tomás, Chile.

${ }^{4}$ Estudiante de Psicología. Facultad de Ciencias Sociales y Comunicaciones, Universidad Santo Tomás, Chile.

${ }^{5}$ Psicólogo. Doctor en Psicoterapia. Docente. Escuela de Psicología, Facultad de Ciencias Sociales y Comunicaciones, Universidad Santo Tomás, Chile. E-mail: cristobalguerra@santotomas.cl

Escuela de Psicología, Facultad de Ciencias Sociales y Comunicaciones, Universidad Santo Tomás, Chile.

\section{Resumen}

El uso de Internet está masificado en casi todo el mundo. Existe evidencia de que su uso excesivo tiene consecuencias negativas, por lo que es necesario contar con instrumentos que evalúen su uso. El Cuestionario de Experiencias Relacionadas con Internet (CERI) es un instrumento de dos factores que evalúan conflictos (intrapersonales e interpersonales) asociados a su uso excesivo. Estudios realizados en distintas partes del mundo han utilizado este instrumento sin analizar sus propiedades psicométricas con la sufi- ciente profundidad y han privilegiado el uso del puntaje total en lugar del puntaje de los dos factores. Por esta razón, en este artículo se evalúan las propiedades psicométricas del instrumento en población chilena. Se realizaron dos estudios en los que participaron un total de 626 estudiantes universitarios (180 en el estudio 1 y 446 en el estudio 2) que respondieron el CERI y un cuestionario de abuso del teléfono móvil. En el estudio 1 se realizó un análisis factorial exploratorio que sugirió seleccionar la estructura unifactorial en lugar de la estructura bifactorial original, aunque ambas presentaban adecuada validez conver- 
gente (relaciones directas con el abuso del teléfono móvil). En el estudio 2 se realizó un análisis factorial confirmatorio y ambas estructuras factoriales presentaron buen ajuste, pero la consistencia interna del factor único fue mayor que la de los dos factores originales. Es necesario continuar evaluando la estructura factorial del CERI en orden a determinar cuál es la mejor alternativa factorial, mientras tanto la estructura unifactorial parece entregar mayores garantías psicométricas.

Palabras clave: Internet; Adicción; Confiabilidad; Validez; Análisis factorial.

\section{Abstract}

A large proportion of the world's population has access to the Internet. On the one hand this has brought undeniable benefits to society by facilitating access to information. But on the other hand, the excessive use of the Internet has also brought with it a series of new problems ranging from physical injuries (for example: tendinitis), psychological pathologies (for example: Internet addiction) to new forms of crime (for example: online sexual abuse). For this reason, it is necessary to have reliable and valid instruments that evaluate the use and abuse of the Internet. The Internet Related Experiences Questionnaire (CERI) has two factors that evaluate conflicts (intrapersonal factor, with 6 items and interpersonal factor, with 4 items) associated with excessive use of the Internet. Studies in different parts of the world (Spain, Chile and other Latin American countries) have used this instrument without analyzing its psychometric properties with sufficient depth. These studies have mostly used the total CERI score (10 items) instead of the two-factor structure, but they have not justified this choice. For this reason, this paper intends to analyze the factorial structure of the instrument, its convergent validity and its internal consistency in the Chilean population. We conducted two researches in which participated a total of 626 university students (180 in the study 1, and 446 in the study 2). The participants responded to the CERI and a questionnaire of abuse of the mobile phone (CERM). In the study 1 , the results of exploratory factor analyzes suggest to use a unifactorial structure which included the 10 items, instead of the original bifactorial structure. In particular, in the bifactorial structure item 1 was not included in any factor (factorial loads less than .2), two items (items 5 and 10) presented similar factor loads in both factors and other two items (items 8 and 9) were included in the factor that it did not correspond. The single factor was greater internal consistency than that of the original intrapersonal and interpersonal factors (Cronbach's alpha of .78, .68 and .56, respectively). Although both alternatives have adequate convergent validity (direct relations with mobile phone abuse). In study 2 , a confirmatory factorial analysis was carried out in order to compare the unifactorial structure obtained in study 1 with the bifactorial structure of the original study. The results show that both factorial structures presented good fit, but again the internal consistency of the single factor was greater than that of the original intrapersonal factor and interpersonal factor (Cronbach's alpha of .74, .61 and .51 respectively). The low reliability of interpersonal and intrapersonal factors may be due to the few number of items grouped in them (4 and 6 respectively). It is necessary to continue evaluating the factorial structure of the CERI in order to determine which is the best factorial alternative or if it is necessary to increase the number of items of interpersonal and intrapersonal factors. Although the results are not conclusive, for the moment, it seems advisable to use the one factor structure as it seems to give further psychometric guarantees.

Keywords: Internet; Addiction; Reliability; Validity; Factor analysis. 


\section{Introducción}

Desde hace algunos años, una amplia proporción de la población mundial tiene acceso a la red, lo que ha traído innegables beneficios a la sociedad (Mayer, 2011). No obstante, el uso excesivo de Internet también ha traído aparejada una serie de nuevas problemáticas que van desde lesiones físicas (ej. tendinitis), patologías psicológicas (ej. adicción a Internet) hasta nuevas formas de delinquir (ej. abuso sexual online), tal como lo han documentado diversos autores (Berner y Santander, 2012; Block, 2008; Quayle y Newman, 2015).

Como en el resto del mundo, la mayoría de la población chilena es usuaria de Internet. Un reciente estudio muestra que el $84.9 \%$ de la población declara utilizar diariamente la red (Pinto y González, 2016). Esta cifra sería mayor en los menores de edad (PUCV, 2017). En particular, el $91 \%$ de niños y adolescentes encuestados reporta que utiliza Internet frecuentemente para hacer tareas escolares, el $80 \%$ lo usa para contactarse con sus redes sociales y el $79 \%$ para jugar en línea.

En la última década se ha puesto en relieve la necesidad de realizar estudios que permitan estimar la magnitud del uso de Internet en la población y conocer los riesgos asociados a ello (Bianchi y Phillips, 2005; Castellana, Sánchez-Carbonell, Graner y Beranuy, 2007; Echeburúa, Labrador y Becoña, 2009; Labrador y Villadongos, 2010). Con ese objetivo, en España se diseñó el Cuestionario de Experiencias Relacionadas con Internet (CERI; Beranuy, Chamarro, Graner y Carbonell, 2009). El instrumento tiene 10 ítems, un formato de autorreporte y alternativas de respuesta en escala Likert de 1 a 4 puntos. Fue validado en Cataluña con una muestra de 1879 estudiantes (de 12 a 25 años), de los cuales 322 eran universitarios y 1557 cursaban la educación secundaria. En el estudio original se evaluaron dos posibles estructuras factoriales (de dos y un factor) mediante un análisis exploratorio. Los autores optaron por retener la estructura de dos factores, que agrupa los ítems relacionados con conflictos intrapersonales (6 ítems; ej. "Cuando tienes problemas, ¿conectarte a Internet te ayuda a evadirte de ellos?") e interpersonales (4 ítems; ej. “¿Piensas que tu rendimiento académico o laboral se ha visto afectado negativamente por el uso de la red?"). Ambos factores presentaron una consistencia interna adecuada (alfa de Cronbach de .81 y .75, respectivamente) y se obtuvieron evidencias de validez convergente (asociaciones directas con una medida de abuso del teléfono móvil y con otra medida de abuso de Internet). Los autores del estudio original español justifican el uso de una medida de abuso al móvil para evaluar la validez convergente de la escala, debido a que tanto Internet como el teléfono móvil son herramientas pertenecientes a las nuevas tecnologías de la información y comunicación que tienen un alto poder de refuerzo para sus usuarios, con el potencial de generar conducta adictiva (Beranuy et al., 2009). Por otro lado, hoy en día, gran parte de los usuarios de Internet se conectan a la web por el teléfono móvil (PUCV, 2017).

Posteriormente, autores de España y Latinoamérica han utilizado el CERI para describir el uso de Internet en distintas poblaciones. Llama la atención que, pese a que los autores del CERI recomiendan la estructura bifactorial por sobre la unifactorial, en todos los estudios revisados, únicamente se utiliza la puntuación total del CERI o se utilizan las puntuaciones por factores, pero haciendo alusión únicamente a la confiabilidad de la escala total. Por ejemplo, en España se encontraron dos estudios que solo consideran los resultados de la puntuación total para describir el uso de Internet en adolescentes y en universitarios (Carbonell, Chamarro, Griffiths, Oberst, Cladellas y Talarn, 2012; Cruces, Guil, Sánchez y Pereira, 2016). En ambos estudios se reporta un alfa de Cronbach de .78 para la escala total de 10 ítems, pero no se dan datos de los dos factores. También en España, de la Villa y Suárez (2016) utilizan 
el CERI. Aunque estos autores únicamente reportan el alfa de la puntuación total (.87), igualmente hacen uso de las puntuaciones de los dos factores.

El caso de Latinoamérica es similar. En Colombia, Redondo, Rangel, Luzardo e Inglés (2016), utilizan solo la puntuación total del instrumento con universitarios, reportando una adecuada consistencia interna $(\alpha=.76)$. En Paraguay, el único estudio encontrado fue realizado con universitarios y reporta la confiabilidad de la escala total $(\alpha=.98)$ y la del factor de conflictos intrapersonales $(\alpha=.96)$; sin embargo, no se hace referencia al factor de conflictos interpersonales, ni se explica la razón de esta omisión (Britos y Brítez, 2015).

En Chile, se encontró un estudio que utiliza el CERI para describir el uso de Internet en una muestra de personas de entre 12 y 30 años (Beranuy, Fernández- Montalvo, Carobonell y Cova, 2016). Estos autores son los únicos que hacen referencia a un proceso de adaptación del instrumento a un contexto cultural diferente al español (modificaron dos palabras y utilizaron una escala Likert en un rango de 1 a 7). No obstante, y pese a las modificaciones realizadas, no evalúan la estructura factorial pertinente en población chilena, ni reportan la consistencia interna del CERI en Chile.

Todos los estudios recién citados utilizan el CERI para describir el uso de Internet en distintas poblaciones y países, sin haber profundizado en la estructura factorial $y$ confiabilidad de las puntuaciones de la escala. Esto sería una imprecisión, ya que los instrumentos no poseen validez universal, por lo que se requiere evaluar sus propiedades psicométricas en los diferentes contextos en los que se utilizan (Oviedo y Campo-Arias, 2005). En particular, estos autores plantean que la confiabilidad de las escalas de medición debe ser evaluada e informada en cada población donde se utilice, de tal manera de tener una estimación del error de medición. De este modo, se hace necesario realizar esfuerzos por evaluar las propiedades psicométricas de nuevos instrumentos antes de su uso masivo
(Contreras, Zalazar-Jaime, De Mier, Aparicio y Cupani, 2016). Es por eso que el presente artículo describe los resultados de dos estudios que han tenido el objetivo de analizar la estructura factorial del instrumento en Chile y ayudar a visibilizar las ventajas o desventajas de utilizar la versión bifactorial o unifactorial. En el primer estudio se realiza un análisis factorial exploratorio de la escala, se evalúa su consistencia interna y se explora su validez convergente -esto último mediante la evaluación de su relación con una media de abuso del teléfono móvil. En el segundo estudio se realiza un análisis factorial confirmatorio y de consistencia interna del CERI.

La relevancia del estudio se relaciona con la necesidad de contar con instrumentos que provean suficientes garantías psicométricas para evaluar un fenómeno reciente, pero de alta relevancia social como lo es el uso excesivo de Internet. El contar con instrumentos válidos y confiables permitirá, no solo estimar la magnitud del problema en poblaciones y sujetos particulares, sino también evaluar la efectividad de programas preventivos o de intervención en personas con uso excesivo de Internet.

\section{Estudio 1}

\section{Participantes}

Participaron 180 estudiantes universitarios de primer año, residentes en Viña del Mar, Chile. La mayoría eran mujeres $(71.1 \%)$ que tenían entre 18 y 30 años $(M=21.01$ años; $D T=3.18)$. Estos estudiantes cursaban las carreras de Técnico en Trabajo Social (60\%), Tecnología Médica (28.3 \%) y Nutrición (11.7\%).

\section{Instrumentos}

\section{Cuestionario de Experiencias Relacionadas con Internet (CERI; Beranuy et al., 2009).}

Se trata de un instrumento autoaplicado de 10 ítems que evalúa el abuso de Internet. 
En su versión inicial posee dos factores: conflictos intrapersonales (ítems 4, 5, 6, 7, 9, 10) y conflictos interpersonales (ítems 1, 2, 3, 8). El puntaje de respuesta varía entre 1 (nada) y 4 (mucho). A mayor puntuación, mayor es la probabilidad de tener un uso problemático de Internet.

\section{Cuestionario de Experiencias Relacionadas con el Móvil (CERM; Beranuy et al., 2009).}

Se trata de un instrumento autoaplicado de 10 ítems que evalúa el uso problemático del teléfono celular. El instrumento está organizado en dos factores (conflictos y uso comunicacional-emocional). El puntaje de respuesta varía entre 1 (nada) y 4 (mucho). A mayor puntuación, mayor es la probabilidad de tener un uso problemático del teléfono celular. En este estudio, el CERM obtuvo los siguientes valores de consistencia interna alfa: .68 para la dimensión conflicto, .60 para uso comunicacional y emocional y .73 para la escala total.

\section{Procedimiento}

Previo a su ejecución, el proyecto fue aprobado por un comité de ética universitario. En primer lugar se realizó una prueba piloto a 10 personas para evaluar la comprensión del instrumento por parte de población chilena. La muestra piloto no sugirió cambios, por lo que se trabajó con la versión original del instrumento. Los datos fueron recogidos por los autores al interior de las salas de clases durante el primer semestre del año 2015. Los participantes dieron su consentimiento informado para formar parte de la muestra. Los formularios completados por ellos fueron traspasados a una base de datos sin incluir ninguna información que permitiera su identificación.

\section{Análisis de datos}

En primer lugar se realizaron análisis descriptivos de los ítems y dimensiones origi- nales del CERI (media, desviación típica, valores mínimos y máximos, asimetría y curtosis). Luego, con el objeto de evaluar si los datos eran susceptibles de un análisis factorial, se calculó el coeficiente Kaiser-Meyer-Olkin (KMO) y el test de esfericidad de Barlett. El número de factores a retener fue determinado en base a un análisis paralelo, que fue realizado con el programa Factor 8.02 (Lorenzo-Seva y Ferrando, 2006).

Posteriormente, considerando la escala de respuestas del CERI, la distribución de sus puntuaciones y siguiendo a los autores del estudio original (Beranuy et al., 2009), se analizó la estructura factorial de la escala mediante un análisis factorial exploratorio con el método de mínimos cuadrados generalizados (GLS) con rotación oblicua. Se utilizó la relación chi-cuadrado/grados de libertad para evaluar la idoneidad del modelo factorial resultante. Según Carmines y McIver (1981), índices entre 1 y 3 indican un buen ajuste de los datos al modelo. Dado que el objetivo de este estudio es ayudar a reconocer la mejor estructura factorial, se comparó la estructura que surgió de este estudio con la bifactorial del estudio de validación original en España. Los datos fueron analizados con el SPSS (Statistical Package for the Social Sciences; IBM Corporation, 2012).

Porúltimo, se analizó la consistencia interna del instrumento mediante el cálculo del coeficiente alfa de Cronbach y también mediante el alfa ordinal (Domínguez-Lara, 2018) y se buscó evidencia de su validez convergente, en base al análisis de su relación con las puntuaciones del CERM (rho de Spearman) en una parte de la muestra $(n=60)$.

\section{Resultados}

La Tabla 1 muestra los descriptivos de cada ítem y de las dimensiones originales del CERI. Luego, dado que los análisis preliminares permiten la factorización de los datos $(\mathrm{KMO}=$ .78; Prueba de esfericidad de Barlett $=331.2$; $g l=45 ; p<.0001)$, se realizó el análisis facto- 
rial exploratorio. Se prefijó la obtención de un solo factor, ya que el análisis paralelo así lo sugirió. El factor resultante permite explicar el $31.64 \%$ de la variabilidad de los ítems y retiene a los 10 ítems de CERI. El modelo presenta un buen ajuste $\left(\chi^{2} / \mathrm{gl}=1.78\right)$. La Tabla 2 muestra las cargas factoriales de los ítems de la solución unifactorial resultante. Respecto a la consistencia interna, el factor único del CERI presenta un valor alfa de Cronbach de .75 y un alfa ordinal de .74 .

Por las razones ya señaladas en la introducción, se decidió comparar la estructura de un factor con la de dos factores obtenida en España. Por esta razón, se repitió el análisis prefijando una solución bifactorial, pero pese a que presenta un buen ajuste $\left(\chi^{2} / \mathrm{gl}=1.32\right)$, esta estructura no coincide con la obtenida en el estudio original. En particular, el ítem 1 no fue incluido en ningún factor (cargas factoriales menores a .2), dos ítems (5 y 10) presentaron cargas factoriales similares en ambos factores y otros dos ítems ( 8 y 9) fueron incluidos en el factor que no correspondía (ver Tabla 2). Dado que la estructura bifactorial no es compatible con la de la versión original, no es posible calcular el alfa ordinal. De todas formas se calculó el alfa de Cronbach de los factores intra e interpersonal, considerando los ítems que debían haber cargado en cada factor, según el estudio original español. El alfa de Cronbach fue de .68 y .56 , respectivamente.

Por último, se aprecia que tanto la puntuación total del CERI como la de sus factores intra e interpersonal se encuentran directamente relacionadas con las tres puntuaciones del CERM. En todos los casos se aprecia que a mayor uso de Internet, mayor es el uso del teléfono celular, lo que ofrece evidencia de la validez convergente de la escala (ver Tabla 3 ).

Tabla 1. Media, desviación típica, valores mínimo y máximo, asimetría y curtosis de los ítems del CERI en el estudio $1(n=180)$.

\begin{tabular}{ccccccc}
\hline Ítem & M & DT & Mín. & Máx. & Asimetría & Curtosis \\
\hline 1 & 1.62 & .82 & 1 & 4 & 1.37 & 1.41 \\
2 & 2.19 & .82 & 1 & 4 & .20 & -.54 \\
3 & 1.83 & .89 & 1 & 4 & .86 & -.08 \\
4 & 1.92 & .84 & 1 & 4 & .44 & -.73 \\
5 & 2.02 & .97 & 1 & 4 & .52 & -.83 \\
6 & 1.73 & .91 & 1 & 4 & 1.09 & .26 \\
7 & 1.59 & .82 & 1 & 4 & 1.44 & 1.53 \\
8 & 1.31 & .55 & 1 & 4 & 1.84 & 3.56 \\
9 & 2.61 & .98 & 1 & 4 & -.01 & -1.02 \\
10 & 1.62 & .81 & 1 & 4 & 1.24 & 1.25 \\
CERI Intra & 11.49 & 3.33 & 6 & 23 & .74 & .37 \\
CERI Inter & 6.94 & 2.05 & 4 & 14 & .71 & .25 \\
CERI Total & 18.43 & 4.69 & 10 & 35 & .78 & .56 \\
\hline
\end{tabular}


Tabla 2. Carga factorial de los items del CERI en la solución unifactorial obtenida en el estudio 1 en comparación a la estructura de dos factores (intrapersonal e interpersonal) propuesta en el estudio original $(n=180)$.

\begin{tabular}{|c|c|c|c|}
\hline \multirow[b]{3}{*}{ Ítem } & \multicolumn{3}{|c|}{ Análisis Factorial Exploratorio } \\
\hline & \multirow{2}{*}{$\begin{array}{c}1 \text { factor } \\
\text { CERI total }\end{array}$} & \multicolumn{2}{|c|}{2 factores } \\
\hline & & Intrapersonal & Interpersonal \\
\hline 1 & .20 & & \\
\hline 2 & .70 & & -.78 \\
\hline 3 & .60 & & -.67 \\
\hline 4 & .53 & .51 & \\
\hline 5 & .45 & .28 & -.23 \\
\hline 6 & .43 & .65 & \\
\hline 7 & .52 & .71 & \\
\hline 8 & .41 & .49 & \\
\hline 9 & .62 & & -.64 \\
\hline 10 & .44 & .28 & -.22 \\
\hline
\end{tabular}

Nota: Solo se incluyen cargas factoriales mayores o iguales a .20. CERI intrapersonal (ítems 4, 5, 6, 7, 9, 10); CERI interpersonal (ítems 1, 2, 3, 8).

Tabla 3. Relaciones (rho de Spearman) entre el CERI y el CERM $(n=60)$.

\begin{tabular}{lccc} 
& CERM conflicto & CERM uso & CERM total \\
\hline CERI total & $.39^{* *}$ & $.46^{* *}$ & $.48^{* *}$ \\
CERI intra & $.35^{* *}$ & $.42^{* *}$ & $.44^{* *}$ \\
CERI inter & $.37^{* *}$ & $.42^{* *}$ & $.44^{* *}$ \\
\hline
\end{tabular}

** $p<.01$

\section{Estudio 2}

\section{Participantes}

Participaron 446 estudiantes universitarios de primer año, residentes en Viña del Mar (Chile). La mayoría eran mujeres $(76.9 \%)$ y sus edades fluctuaban entre los 18 y 31 años $(M=20.36 ; D T=2.48)$. Las carreras con mayor representación fueron Psicología (21.1\%), Enfermería (16.4\%), Medicina Veterinaria (14.6\%), Kinesiología (11.9\%) Fonoaudiología $(8.3 \%)$, Tecnología Médica $(8.3 \%)$ y Nutrición $(8.1 \%)$. 


\section{Instrumento}

Cuestionario de Experiencias Relacionadas con Internet (CERI; Beranuy et al., 2009), ya descripto en el estudio 1.

\section{Procedimiento}

El procedimiento fue el mismo que en el estudio 1. Los datos fueron recolectados durante el primer semestre del año 2016.

\section{Análisis de datos}

En este estudio se realizó un análisis factorial confirmatorio tendiente a evaluar el ajuste de los datos a la solución unifactorial encontrada en el estudio 1 y a la estructura bifactorial del estudio de validación original. Siguiendo a Li (2016), el análisis confirmatorio fue realizado con el método para datos ordinales denominado mínimos cuadrados diagonales (WLSMV). Se consideró un buen ajuste en base al estadígrafo de chi cuadrado. Aunque tradicionalmente se sugiere considerar un buen ajuste cuando el valor de este estadígrafo no es significativo $(p>.05)$, es sabido que existe una tendencia a resultar significativo en muestras grandes (Fujikoshi, 2000). De este modo, al igual que en el estudio 1, se consideró la razón chi cuadrado/grados de libertad como indicador de ajuste (valores esperados entre 1 y 3). Siguiendo a Schumacker y Lomax (2004), también se consideró un buen ajuste en base a un RMSEA $\leq .08$, CFI $\geq .90$ y TLI $\geq .90$. Luego, con el objeto de poder comparar el ajuste de los modelos de uno y dos factores, se repitió el análisis con el método de máxima verosimilitud robusto (MLR), el cual arroja el índice de AKAIKE (AIC) que permite comparar a los dos modelos, debiendo privilegiarse el modelo con un menor AIC. Los datos fueron analizados con MPlus (Muthen, L. y Muthen, B., 2012).

\section{Resultados}

En la Tabla 4 se pueden observar los estadísticos descriptivos para todos los ítems del CERI en el estudio 2. Respecto al análisis factorial se compara la solución unifactorial con la bifactorial del estudio original.

Tabla 4. Media, desviación típica, valores mínimo y máximo, asimetría y curtosis de los ítems del CERI en el estudio $2(n=446)$.

\begin{tabular}{ccccccc}
\hline Ítem & $M$ & $D T$ & Mín. & Máx. & Asimetría & Curtosis \\
\hline 1 & 1.58 & .68 & 1 & 4 & 1.03 & .83 \\
2 & 2.26 & .74 & 1 & 4 & .13 & -.31 \\
3 & 2.07 & .84 & 1 & 4 & .52 & -.24 \\
4 & 2.12 & 1.00 & 1 & 4 & .55 & -.76 \\
5 & 2.01 & 1.01 & 1 & 4 & .55 & -.91 \\
6 & 1.79 & .89 & 1 & 4 & .98 & .19 \\
7 & 1.45 & .72 & 1 & 4 & 1.79 & 3.14 \\
8 & 1.34 & .61 & 1 & 4 & 1.89 & 3.62 \\
9 & 2.92 & .93 & 1 & 4 & -.31 & -.91 \\
10 & 1.77 & .82 & 1 & 4 & .83 & .11 \\
CERI Intra & 12.06 & 3.2 & 6 & 24 & .56 & .17 \\
CERI Inter & 7.24 & 1.8 & 4 & 14 & .46 & -.09 \\
CERI Total & 19.31 & 4.5 & 10 & 36 & .55 & .14 \\
\hline
\end{tabular}


Los resultados que arroja el análisis con el estimador WLSMV muestran un buen ajuste de los datos al modelo unifactorial. Aunque el índice de chi cuadrado es significativo, el índice chi cuadrado/grados de libertad está dentro del rango recomendado $\left(\chi^{2}=60.141\right.$; $\left.\mathrm{gl}=35 ; p<.01 ; \chi^{2} / \mathrm{gl}=1.72\right)$. Los otros índices apoyan el ajuste del modelo (RMSEA $=.04$;
$\mathrm{CFI}=.97$; TLI $=.97)$. La figura 1 muestra las cargas factoriales de cada ítem en el factor, todas ellas son mayores a .3. Los resultados con el estimador MLR son similares, mostrando un IAC de 9438.330. El alfa de Cronbach del factor único, en esta muestra, fue de .72 , en tanto que el alfa ordinal fue de .78 .

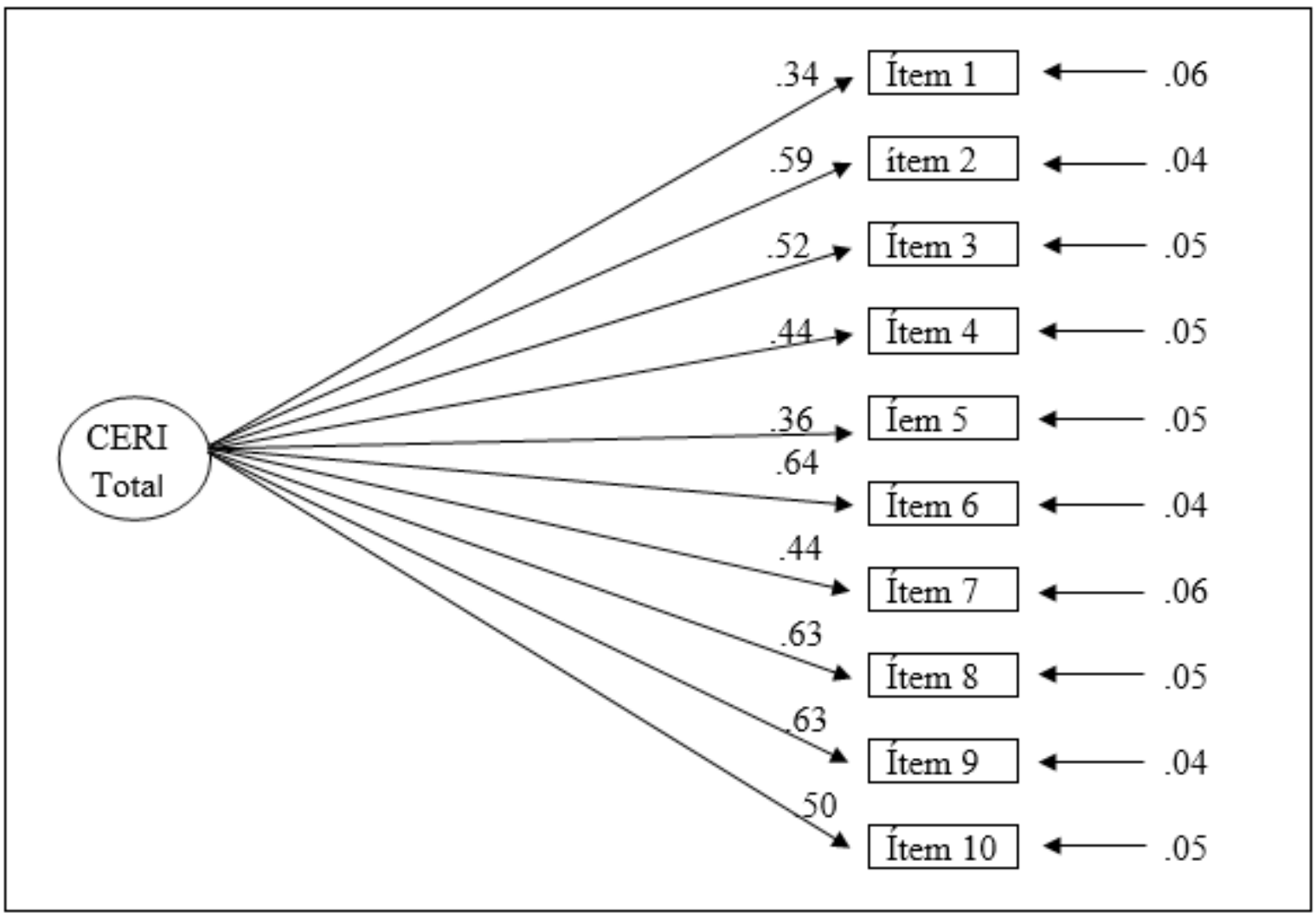

Figura 1. Cargas factoriales (y error) de los ítems en la solución unifacorial en el estudio 2 $(n=446)$.

La figura 2 muestra las cargas factoriales del modelo de dos factores sugerido en el estudio original español. Se aprecia que los ítems cargan de forma satisfactoria en su respectivo factor y que ambos factores presentan una elevada correlación entre sí. El modelo ajusta de forma similar al modelo unifactorial $\left(\chi^{2}=57.771 ; \mathrm{gl}=34 ; p<.01 ; \chi^{2} / \mathrm{gl}=1.70 ;\right.$
RMSEA $=.04 ; \mathrm{CFI}=.97 ; \mathrm{TLI}=.97) . \mathrm{El} \mathrm{IAC}$ obtenido en base al análisis con MLR fue levemente mejor que el modelo unifactorial $(\mathrm{IAC}=9437.032)$, pero la consistencia interna del factor intrapersonal e interpersonal fue menor que el del factor único (alfa de Cronbach de .61 y .51 y alfa ordinal de .67 y .64 , respectivamente). 


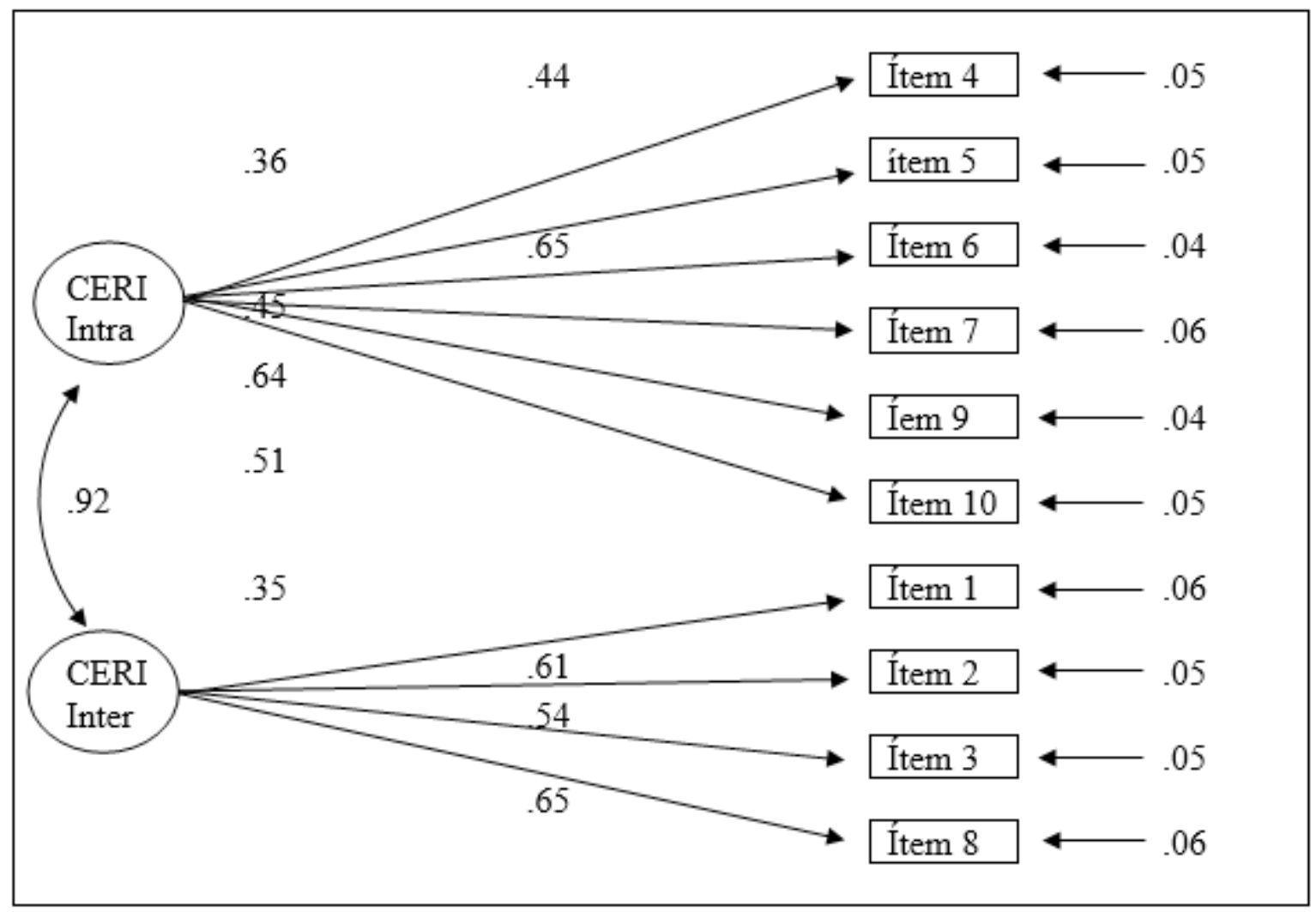

Figura 2. Cargas factoriales (y error) de los ítems en la solución bifacorial en el estudio 2 $(n=446)$.

Considerando que las dos estructuras factoriales presentan un ajuste similar y que el factor intrapersonal presenta una alta correlación con el interpersonal, se decidió testear un tercer modelo en el que los dos factores de primer orden (CERI interpersonal y CERI intrapersonal) se explicaran desde un factor de segundo orden (CERI total). En base al planteamiento de Geiser (2013), este modelo fue desechado debido a parámetros fuera de rango en el factor interpersonal. La varianza residual de este factor fue negativa (varianza residual $=-.13$ ), lo que puede deberse, según este autor, a la existencia de pocos ítems.

\section{Discusión}

El presente artículo tuvo como objetivo analizar la estructura factorial del CERI en población chilena, su consistencia interna y su validez convergente. La relevancia del estudio tiene que ver con la necesidad de utilizar medidas confiables y válidas de los constructos de importancia social (Oviedo y Campo-Arias, 2005), como es actualmente el abuso de Internet. A pesar del frecuente uso que se hace del CERI, a casi 10 años de su creación, aún no hay estudios que revisen sus propiedades psicométricas.

Respecto a la estructura factorial del instrumento, los resultados del estudio 1 presentan algunas diferencias con el estudio de validación original (Beranuy et al., 2009), pues sugiere la conservación de una estructura de un solo factor.

La estructura bifactorial no es recomendada por los resultados del análisis factorial del primer estudio, ya que hubieron dos ítems que cargaron en el factor opuesto a lo esperado. Se trata del ítem 8 ("Cuando no estás conectado a Internet, ¿te sientes agitado o preocupado?") que, según el estudio de validación original, debiera cargar en el factor interpersonal, pero en este estudio carga en 
el intrapersonal. Al revisar el contenido del ítem parece más coherente el resultado de este estudio, ya que se alude a sintomatología ansiosa de carácter intrapersonal. Algo similar ocurre con el ítem 9 ("Cuando navegas por Internet, el tiempo pasa sin darte cuenta") que debiera cargar en el factor intrapersonal, pero lo hace en el interpersonal. Si bien desde el análisis del contenido del ítem se entiende que los autores lo hayan nominado como un indicador intrapersonal, se entiende también que haya cargado en el factor interpersonal, por los conflictos que el excesivo tiempo dedicado a Internet pudiese tener a nivel interpersonal (familiar, académico o laboral), tal como sugiere Fernández-Sánchez (2013).

Pese a lo anterior, tal como en el estudio de validación original (Beranuy et al., 2009), los resultados entregan evidencia preliminar de validez convergente de todas las puntuaciones del CERI, dejando nuevamente abierta la utilidad de una estructura de dos factores. Tanto el CERI total como sus dimensiones intra e interpersonal se asociaron directamente a una medida de abuso del teléfono móvil. Esta relación puede ser considerada como una evidencia preliminar de validez convergente, ya que en ambos instrumentos se está midiendo el uso de nuevas tecnologías de la información que, llevadas a un extremo, pueden dar pie a un uso abusivo (Sánchez-Carbonell, Beranuy, Castellana, Chamarro y Oberst, 2008). No obstante, debe considerarse que en este estudio se evaluó esta relación con una pequeña proporción de la muestra $(n=60)$, lo que sugiere ser cautos a la hora de proyectar los alcances de esta relación. Futuros estudios deberían hacer mayores esfuerzos por encontrar evidencia más sólida de la validez convergente de la escala.

Adicionalmente, el análisis factorial confirmatorio respalda tanto el uso de una estructura de un factor como de dos factores. Ambas soluciones presentan un ajuste similar e incluso el AIC de la solución de dos factores es mejor que el de uno solo. No obstante, hay dos argumentos que abogan en favor de la estructura unifactorial. Primero, se aprecia una elevada correlación entre los dos factores, lo que sugiere que una solución más parsimoniosa podría ser la de un solo factor. Segundo, se aprecian problemas de confiabilidad en los factores del CERI, sobre todo en el factor interpersonal. En cambio, la puntuación total presenta adecuados índices de consistencia interna, tal como sucedió en el estudio 1 (Guilford y Fruchter, 1984).

La baja confiabilidad del factor interpersonal probablemente esté siendo ocasionada por el escaso número de ítems del factor (solo 4). Lloret-Segura, Ferreres-Traver, Hernández-Baeza y Tomás-Marco (2014) sugieren utilizar más de 4 ítems para no comprometer la consistencia interna de la medida. Hubiera sido interesante conocer la fiabilidad de los factores en los diferentes estudios realizados en Chile (Beranuy et al., 2016) y en el resto del mundo (Britos y Brítez, 2015; Carbonell et al., 2012; Cruces et al., 2016; de la Villa y Suárez, 2016; Redondo et al., 2016) para compararla con la obtenida en este estudio, pero ninguno realizó un análisis acabado de la confiabilidad de los factores y solo se cuenta con el reporte del estudio inicial (Beranuy et al., 2009). Se considera necesario continuar monitoreando la estructura factorial y la consistencia interna de los factores del CERI en orden a determinar, en el futuro, la necesidad de incluir nuevos ítems que entreguen mayor variabilidad a la dimensión intra e interpersonal y, por esa vía, mejoren su consistencia interna.

Aunque no se puede descartar la pertinencia de la estructura de dos factores, los resultados de ambos estudios son consistentes respecto de la utilidad de la solución de un solo factor. Esta solución aporta evidencia de validez de constructo, factorial y de consistencia interna en los dos estudios aquí presentados. Esto es coherente con el hecho de que la estructura de un factor ha sido la privilegiada por los investigadores en distintas partes del mundo, lo que da cuenta también de su utilidad práctica (Carbonell et al., 2012; de la Villa y Suárez, 2016; Redondo et al., 2016). 
Respecto de la estructura original de dos factores, los resultados son menos concluyentes. Mientras en el estudio 1 no se apoya esta solución dejando entrever algunos problemas de validez en el contenido de los ítems, en el estudio 2 esta estructura presenta buen ajuste, pero una baja confiabilidad. Como ya se ha dicho, es necesario continuar analizando las propiedades psicométricas de la escala en orden a informar a los investigadores sobre las ventajas y desventajas del uso de una solución o de la otra.

Hay que considerar dos posibles explicaciones que pueden ayudar a entender la diferencia de estructura factorial recomendada en este artículo en comparación al original, más allá de las diferencias culturales que pueden existir entre Chile y España. Primero, el estudio original fue realizado hace 10 años. Aunque el tiempo transcurrido no es excesivo para la mayoría de las problemáticas sociales, sí podría serlo en el caso de Internet, donde el comportamiento del usuario tiende a variar considerablemente al paso de algunos años (Garza, 2012). Parece necesario entonces que, cada cierto tiempo se vuelvan a revisar las propiedades psicométricas de instrumentos que evalúan conflictos dinámicos como es el uso de Internet. Segundo, el estudio español fue realizado mayoritariamente con estudiantes de secundaria $(M=15.52$ años; $D T=2.43$ ) en tanto que el presente estudio fue realizado con estudiantes universitarios $(M=20.54$ años; $D T=2.70)$. Los estudios precedentes dan cuenta de diferencias atribuibles a la edad en el riesgo de abusar de Internet, donde se destaca la especial vulnerabilidad de los adolescentes por sobre otros grupos etarios (Bianchi y Phillips, 2005; Madell y Muncer, 2004). De hecho, en el mismo estudio original se observan diferencias significativas en las puntuaciones del CERI entre menores y mayores de edad (Beranuy et al., 2009). Una de las limitaciones de este estudio es que únicamente trabajó con universitarios, por lo que queda pendiente realizar estudios con adolescentes que permitan evaluar la inva- rianza de la estructura factorial en distintos grupos etarios.

Dicho esto, se considera que es posible utilizar el CERI en población chilena. Sin embargo, por el momento, se sugiere privilegiar el uso de la puntuación total por sobre las puntuaciones de los factores intra e interpersonales, ya que ésta otorga mayores garantías psicométricas. Futuros estudios deberían continuar analizando la estructura factorial más recomendada en distintos tramos etarios y los puntos de corte que permitan diferenciar un uso normal de un uso excesivo del Internet.

\section{Referencias bibliográficas}

Beranuy, M., Chamarro, A., Graner, C., y Carbonell, X. (2009). Validación de dos escalas breves para evaluar la adicción a Internet y el abuso de móvil. Psicothema, 21(3), 480-485.

Beranuy, M., Fernández- Montalvo, J., Carobonell, X. y Cova, F. (2016). Características del uso de Internet en los cibercafés. Terapia Psicológica, 34(1), 5-14. doi: 10.4067/S071848082016000100001

Berner, G.J. y Santander, J. (2012). Abuso y dependencia de Internet: la epidemia y su controversia. Revista Chilena de Neuropsiquiatría, 50(3), 181-190. doi: 10.4067/S071792272012000300008

Bianchi, A. y Phillips, J.G. (2005). Psychological predictors of problem mobile phone use. Cyberpsychology \& Behavior, 8(1), 39-51. doi: 10.1089/cpb.2005.8.39

Block, J. (2008). Issues for DSM-V: Internet addiction. American Journal of Psychiatry, 165(3), 306-307. doi: 10.1176/appi.ajp.2007.07101556

Britos, E.M. y Brítez, R. (2015). Nuevas tecnologías y juventud: Uso y acceso a Internet, móvil $\mathrm{y}$ redes sociales en universitarios de ambos sexos, de una carrera de humanidades (psicología), de una universidad privada, período 2014. Revista Cientifica de la UCSA, 2(2), 63-74.

Carbonell, X., Chamarro, A., Griffiths, M., Oberst, U., Cladellas, R. y Talarn, A. (2012). Problematic Internet and cell phone use in Spanish 
teenagers and young students. Anales de Psicología, 28(3), 789-796. doi: 10.6018/ analesps.28.3.156061

Carmines, E. y McIver, J. (1981). Social measurement: Current issues. En G. Bohrnstedt y E. Borgatta (Eds.), Analyzing models with unobserved variables: Analysis of covariance structures (65-115). Beverly Hills, CA: Sage.

Castellana, M., Sánchez-Carbonell, X., Graner, C. y Beranuy, M. (2007). El adolescente ante las tecnologías de la información y la comunicación: Internet, móvil y videojuegos. Papeles del Psicólogo, 28(3), 196-204.

Cruces, S., Guil, R., Sánchez, N. y Pereira, J. (2016). Consumo de nuevas tecnologías y factores de personalidad en estudiantes universitarios Commons: Revista de Comunicación y Ciudadanía Digital, 5(2), 203-228. doi: 10.25267/COMMONS.2016.v5.i2.09

Contreras, M., Zalazar-Jaime, M., De Mier, M., Aparicio y Cupani, M. (2016). Escala de Apoyo Parental: Nuevos estudios de estructura interna y su relación con variables socioeconómicas. Interdisciplinaria, 33(2), 299-313. doi: 10.16888/interd.2016.33.2.7

de la Villa, M. y Suárez, C. (2016). Factores de riesgo en el uso problemático de Internet y del teléfono móvil en adolescentes españoles. Revista Iberoamericana de Psicología y Salud, 7, 69-78. doi: 10.1016/j.rips.2016.03.001

Domínguez-Lara, S. (2018). Fiabilidad y alfa ordinal. Actas Urológicas Españolas. 42. 140-141. doi: 10.1016/j.acuro.2017.07.002

Echeburúa, E., Labrador, F.J. y Becoña, E. (2009). Adicción a las nuevas tecnologías. Madrid: Pirámide.

Fernández-Sánchez, N. (2013). Trastornos de conducta y redes sociales en Internet. Salud Mental, 36, 521-527. doi: 10.17711/SM.01853325.2013 .063

Fujikoshi y. (2000). Transformations with improved chi-squared approximations. Journal of Multivariate Analysis, 72, 249-263. doi: 10.1006/jmva.1999.1854

Garza, E. (2012). Uso y Consumo de Internet en Jóvenes Estudiantes: Análisis del Estado de Tamaulipas (Tesis de Doctorado no publicada).
Facultad de Ciencias de la Educación, Universidad de Santiago de Compostela, España.

Geiser, C. (2013). Data Analysis with Mplus. Guilford Press: New York.

Guilford, J.P. y Fruchter, B. (1984). Estadistica aplicada a la psicología y la educación. México, D.F.: McGraw Hill.

IBM Corporation (2012). IBM SPSS Statistics for Windows, Version 21.0. Armonk, NY: IBM Corp.

Labrador, F.J. y Villadongos, S. (2010). Menores y nuevas tecnologías: conductas indicadoras de posible problema de adicción. Psicothema, 22(2), 180-188.

Li, C.H. (2016). Confirmatory factor analysis with ordinal data: Comparing robust maximum likelihood and diagonally weighted least squares. Behaviour Research Methods, 48(3), 936-49. doi: 10.3758/s13428-015-0619-7.

Lloret-Segura, S., Ferreres-Traver, A., Hernández-Baeza, A. y Tomás-Marco, I. (2014). El análisis factorial exploratorio de los ítems: una guía práctica, revisada y actualizada. Anales de Psicología, 30(3), 1151-1169. doi: 10.6018/ analesps.30.3.199361

Lorenzo-Seva, U. y Ferrando, P. (2006). FACTOR: A computer program to fit the exploratory factor analysis model. Behavior Research Methods, 38(1), 88-91. doi: 10.3758/BF03192753

Madell, D. y Muncer, S. (2004). Back from the beach but hanging on the telephone? English adolescent's attitudes and experiences of mobile phones and the Internet. Cyberpsychology \& Behavior, 7, 359-367. doi: 10.1089/1094931041291321

Mayer, M. (2011). La utilización de Internet entre los adolescentes, riesgos y beneficios. Atención Primaria, 43(6), 287-288. doi: 10.1016/j. aprim.2010.12.004

Muthen, L.K. y Muthen, B.O. (2012). Mplus User's Guide. Seventh edition. Los Angeles, CA: Muthen y Muthen.

Oviedo, H.C. y Campo-Arias, A. (2005). Aproximación al uso del coeficiente alfa de Cronbach. Revista Colombiana de Psiquiatría, 34, 572-580.

Pinto, M. y González, B. (2016). Séptima encuesta de acceso, usos y usuarios de Internet. Recupe- 
rado de http://www.subtel.gob.cl/wp-content/ uploads/2015/04/Informe-VII-Encuesta-deAcceso-Usos-y-Usuarios-de-Internet_VF.pdf

PUCV (2017). Estudio PUCV revela hábitos de navegación y consumo digital de los niños y adolescentes del país. Recuperado de http:// pucv.cl/pucv/noticias/destacadas/estudio-pucv-revela-habitos-de-navegacion-y-consumo-digital-de-los-ninos/2017-04-10/131926. html

Quayle, E. y Newman, E. (2015). The role of sexual images in online and offline sexual behaviors with minors. Current Psychiatry Reports, 17(6), 43. doi: 10.1007/s11920-0150579-8.

Redondo, J., Rangel, K., Luzardo, M., e Inglés, C. (2016). Experiencias relacionadas con el uso de Internet y celular en una muestra de estudiantes universitarios colombianos. Revista Virtual Universidad Católica del Norte, 49, 7-22.

Samper-García, P., Mesurado, B., Richaud, M. y Llorca, A. (2016). Validación del Cuestionario de Conciencia Emocional en adolescentes españoles. Interdisciplinaria, 33(1), 163- 176. doi: 10.16888/interd.2016.33.1.10

Sánchez-Carbonell, X., Beranuy, M., Castellana, M., Chamarro, A. y Oberst, U. (2008). Internet and cell phone addiction: passing fad or disorder? Adicciones, 20(2), 149-159.

Schumacker, R.E. y Lomax, R.G. (2004). A beginner's guide to structural equation modeling (2nd Ed.). Mahwah, NJ: Lawrence Erlbaum Associates, Inc.

Recibido: 20 de octubre de 2017 Aceptado: 5 de septiembre de 2019 